\title{
Intervention territoriale visant à lutter contre la pollution atmosphèrique et équité en matière de santé
}

Wahida Kihal, Cindy Padilla et Séverine Deguen

\section{OpenEdition}

Édition électronique

URL : http://journals.openedition.org/rfst/464

DOI : $10.4000 /$ rfst.464

ISSN : 2492-3672

Éditeur

Espaces et SOciétés (UMR 6590)

Référence électronique

Wahida Kihal, Cindy Padilla et Séverine Deguen, «Intervention territoriale visant à lutter contre la pollution atmosphèrique et équité en matière de santé », Revue francophone sur la santé et les territoires [En ligne], Miscellanées, mis en ligne le 09 octobre 2015, consulté le 06 avril 2021. URL : http:// journals.openedition.org/rfst/464 ; DOI : https://doi.org/10.4000/rfst.464

Ce document a été généré automatiquement le 6 avril 2021.

La Revue francophone sur la santé et les territoires est mise à disposition selon les termes de la Licence Creative Commons Attribution - Pas d'Utilisation Commerciale - Partage dans les Mêmes Conditions 4.0 International. 


\title{
Intervention territoriale visant à lutter contre la pollution atmosphèrique et équité en matière de santé
}

\author{
Wahida Kihal, Cindy Padilla et Séverine Deguen
}

\section{Introduction}

\section{Les interventions territoriales et les enjeux environnementaux}

1 Depuis la prise de conscience des enjeux environnementaux dans les années 1970, les interventions publiques visant directement ou indirectement à protéger l'environnement se sont multipliées et le cadre juridique de ces actions s'est très largement développé, particulièrement au niveau européen. Notre quotidien est ainsi largement marqué par cette problématique, dont toutes les échelles territoriales se saisissent comme l'illustre l'implication des différentes collectivités: l'Union Européenne, l'État ainsi que les collectivités territoriales (régions, départements, communes, intercommunalités). Chacune élabore et met en place, dans ses domaines de compétence, des programmes d'action et des interventions en faveur de l'environnement : règlements et directives européennes, lois et règlements nationaux, Agendas 21 nationaux et locaux, plans climat, plans de protection de l'atmosphère et initiatives locales.

2 Un des aspects de la protection de l'environnement se fait de plus en plus prégnant, particulièrement en milieu urbain : il s'agit de la lutte contre la pollution de l'air, véritable enjeu de santé publique. En effet, les effets sanitaires d'une exposition à la pollution de l'air sont aujourd'hui solidement établis: diminution de la capacité respiratoire, inflammation des poumons, asthme, augmentation de la vulnérabilité aux infections respiratoires, et décès prématurés (Rapport AFSSET, 2007). 
3 L'émotion suscitée dans les médias par le pic de pollution intervenu aux mois de mars 2014 et 2015 dans plus de trente départements français témoigne d'une prise de conscience collective de l'importance de lutter contre cette pollution atmosphérique. Plusieurs interventions des collectivités territoriales ayant pour objectif principal (effet direct) ou pour objectif secondaire (effet indirect) de lutter contre la pollution atmosphérique sont mises en place; le bonus/malus écologique, le vélo en libre-service, la limitation de la vitesse de circulation et la circulation alternée ou bien la prise en compte des questions environnementales en matière d'urbanisme.

\section{A qui profitent réellement ces dispositifs?}

4 Si le lien statistique entre un évènement de santé et le niveau de défaveur socioéconomique est bien documenté depuis de nombreuses années (Ferland et al., 1995), les quelques observations empiriques concernant l'effet des programmes de santé publique tendent à montrer que le bénéfice de ceux-ci est également socialement structuré (PNNS - Note de veille, 2010). Ainsi, des interventions à caractère universel (conduite en population générale) se révèlent bénéficier davantage à une catégorie sociale qu'à une autre, ou bien avoir pour conséquence de faire supporter des effets collatéraux à des populations socio-économiquement défavorisées.

5 Peu d'études évaluent systématiquement les effets des interventions de santé publique en fonction des strates sociales. Afin d'illustrer les impacts socialement différenciés que pourrait avoir une intervention visant à diminuer l'exposition des populations aux polluants atmosphériques, les conséquences de la mise en place à Londres du LEZ « Low Emission Zone» ont été analysées par Benmarhnia et al. (Benmarhnia et al. en 2014). Cette intervention consiste en l'application d'un tarif à l'entrée de la zone délimitée pour les véhicules ne respectant pas les normes européennes d'émission, afin de les dissuader d'y circuler. Les auteurs concluent que cette intervention pourrait modifier la répartition spatiale de la pollution atmosphérique au détriment des populations plus vulnérables (Benmarhnia et al., 2014).

6 En France, le programme de recherche Equit'Area ( http://www.equitarea.org) explore les inégalités sociales et territoriales des nuisances environnementales, et leurs évolutions dans le temps. Ces travaux conduits dans quatre agglomérations françaises, Paris, Lyon, Marseille et Lille et décrits plus en détails dans le rapport de l'évaluation du second Plan National Santé Environnement (PNSE2), montrent que durant la décennie 2000, les disparités d'exposition entre les IRIS (Ilot Regroupé pour l'Information Statistique, échelle infra-communale définie par l'INSEE) favorisés et défavorisés se sont réduites à Lille alors qu'elles se sont légèrement aggravées à Lyon (Tableau 1). Ainsi, concernant l'évaluation du PNSE2, le rapport du Haut Conseil de Santé Publique concluait que "à Lille comme dans d'autres agglomérations, bien que les émissions soient globalement à la baisse notamment pour les oxydes d'azote (NOx), la stagnation de l'exposition aux particules et au NO2 pour une fraction importante de la population nationale au cours de toute la décennie 2000 est la marque d'un certain échec de la gestion des zones urbaines denses à la fois en émissions (notamment du trafic routier) et en population » (Rapport du HCSP, 2013).

7 Dans ce contexte, Il apparait alors primordial de s'interroger sur les conséquences involontaires que peuvent avoir la mise en place de ces interventions territoriales sur les inégalités sociales de santé (ISS). 


\section{L'équité en matière de santé au cœur des enjeux environnementaux}

8 Aujourd'hui, les constructions socio-territoriales contribuent à la persistance voire à l'augmentation des inégalités de santé (Deguen et al., 2010). Au travers de deux mécanismes agissant de manière indépendante ou combinée, les populations défavorisées pourraient souffrir de plus forts effets sanitaires de facteurs de risque environnementaux. En effet, les populations modestes pourraient être à la fois soumises à des niveaux plus élevés d'expositions environnementales et/ou pourraient cumuler une diversité de sources d'expositions et de nuisances plus importante. Ces mêmes groupes de population pourraient être également plus sensibles aux effets sanitaires des différentes nuisances environnementales (« différentiel de vulnérabilité ») (O’Neil et al., 2003).

Tableau 1 : Evolution des concentrations moyennes du NO${ }_{2}\left(\mu \mathrm{g} / \mathrm{m}^{3}\right)$ dans des secteurs contrastés sur le plan socio-économique dans l'agglomération de Lyon, entre 2002 et 2009

\begin{tabular}{|c|c|c|c|c|c|c|c|}
\hline \multicolumn{2}{|c|}{ Période } & \multirow{2}{*}{\multicolumn{2}{|c|}{$\begin{array}{l}\text { Concentrations } \mathrm{NO}_{2} \\
\text { dans les IRIS P80 à }\end{array}$}} & \multirow{2}{*}{\multicolumn{2}{|c|}{$\begin{array}{l}\text { Concentrations } \mathrm{NO}_{2} \\
\text { dans les IRIS P2O à }\end{array}$}} & \multirow{3}{*}{$\begin{array}{l}\text { Evolution } \\
\text { (\%) P80 }\end{array}$} & \multirow{3}{*}{$\begin{array}{l}\text { Evolution } \\
\text { (\%) P20 }\end{array}$} \\
\hline \multirow[t]{2}{*}{$\mathrm{T} 1$} & \multirow[t]{2}{*}{ T3 } & & & & & & \\
\hline & & $\mathrm{T} 1{ }^{*}$ & T3* & T1* & T3* & & \\
\hline $\begin{array}{l}2002- \\
2004\end{array}$ & $\begin{array}{l}2007- \\
2009\end{array}$ & $\begin{array}{r}43.8 \\
\pm 4.9\end{array}$ & $\begin{array}{l}36.9 \\
\pm 5.6\end{array}$ & $\begin{array}{l}40.5 \\
\pm 5.0\end{array}$ & $\begin{array}{l}32.6 \\
\pm 5.2\end{array}$ & $-15.9 \%$ & $-19.5 \%$ \\
\hline
\end{tabular}

* (PSO): soème percentile de la distribution de líndice de défaveur socioéconomique, correspondant aux IRIS les plus défavorisés

* (P20): 20ème percentile de la distribution de l'indice de défaveur socioéconomique, correspondant aux IRIS les plus favorisés

* T1: Le premier tercile du calendrier (T1, 2002-2004) et T3: le dernier tercile du calendrier (T3, 2007-2009)

* (P80): 80ème percentile de la distribution de l'indice de défaveur socioéconomique, correspondant aux IRIS les plus défavorisés

* (P20): 20ème percentile de la distribution de l'indice de défaveur socioéconomique, correspondant aux IRIS les plus favorisés

* T1: Le premier tercile du calendrier $(T 1,2002-2004)$ et T3: le dernier tercile du calendrier (T3, 2007-2009)

Source : Étude Equit'Area, Rapport HCSP, 2013

9 En effet, de nombreuses études européennes rapportent que les populations à faible statut socioéconomique sont plus exposées aux polluants atmosphériques. Mais, il ne s'agit pas d'une situation générale puisque qu'en France, une étude a démontré au contraire que les classes moyennes résidant à Strasbourg étaient les plus exposées à la pollution issue du trafic routier (Havard et al., 2009), situation retrouvée à Lyon (Padilla et al.,2013). Ces contrastes tiennent à l'histoire de la construction urbaine, propre à chaque zone géographique investiguée. Quant au différentiel de vulnérabilité, les travaux existants s'accordent à dire que, même lorsque les quartiers les plus défavorisés ne sont pas les plus affectés par une pollution, les conséquences sanitaires de cette exposition y sont plus marquées (Sexton et al., 1997; Rios et al., 2009). Cette plus grande vulnérabilité résulterait notamment d'un état de santé souvent plus dégradé, de conditions de vie plus difficiles et d'un plus faible recours aux soins.

10 Les inégalités de santé sont liées à l'organisation, la gestion et la pratique de l'espace (urbain, péri-urbain ou rural), et marquées par une segmentation directement liée à la valeur foncière du terrain, du logement et du bâti. Ainsi, sauf sous l'effet de politiques 
volontaristes de mixité sociale, des îlots socialement contrastés se créent. Ces derniers dérivent progressivement vers la ségrégation, voire l'invisibilité sociale (Le Blanc, 2009) en raison des inégales capacités des groupes sociaux à porter et faire valoir leurs intérêts.

11 Ainsi, les enjeux de l'équité en matière de santé se situent à l'interface de problématiques environnementales, sociales et territoriales. Face à un tel constat, et alors que les ISS sont une préoccupation majeure en santé publique, la question soulevée ici est la suivante «les bénéfices sur la santé des interventions visant à diminuer la pollution de l'air en milieu urbain diffèrent-ils entre des populations aux caractéristiques socioéconomiques différentes? ». On interroge alors l'existence d'une prise en compte de l'équité en matière de santé, des interventions sur la pollution de l'air

Pour approcher cette question, nous avons observé par des entretiens auprès d'un panel de professionnels et acteurs politiques impliqués, l'enjeu de l'équité en matière de santé dans l'élaboration et la mise en œuvre des interventions territoriales. Plus précisément, au vu de la littérature sur ce sujet, le postulat est que très peu d'études s'intéressent à ce jour à évaluer l'amélioration de l'état de santé grâce à ces interventions et que celles-ci ne vont pas jusqu'à analyser les inégalités qui peuvent en résulter du fait d'une répartition géographique inégale des populations selon leurs caractéristiques socio-économiques.

Nous avons donc cherché à identifier et à expliquer les points de blocage qui empêcheraient de telles démarches en nous entretenant avec différents acteurs impliqués : décideurs publics, spécialistes de l'évaluation des politiques publiques et chercheurs.

Pour répondre à ces interrogations, suite à une présentation de notre méthodologie, nous dresserons dans un premier temps le bilan des actions emblématiques faites sur l'agglomération de Lyon au regard des profils socio-économiques observés; Dans un second temps, nous développerons les facteurs identifiés comme participant au défaut d'évaluation de l'équité des interventions, pour exposer dans un dernier point des propositions qui permettraient de dépasser les obstacles et d'intégrer la préoccupation de l'équité en matière de santé à chaque étape de la lutte contre la pollution de l'air.

\section{Matériel \& méthode}

L'analyse des entretiens visera à discuter des freins identifiés à la prise en compte de l'équité en matière de santé dans la planification des interventions et à la mise en place d'une démarche d'évaluation, en s'entretenant avec différents acteurs impliqués: décideurs publics, spécialistes de l'évaluation des politiques publiques et chercheurs.

Plus particulièrement, la grille d'entretien se structure autour de trois axes principaux :

1. Quelles sont les actions et politiques publiques mises en œuvre visant à diminuer la pollution atmosphérique?

2. Existe-il une évaluation de ces actions au regard de leurs impacts sur la santé et sur les ISS ?

3. Quelles recommandations pourraient être formulées pour qu'une telle évaluation puisse être mise en œuvre?

17 Cette étude qualitative s'est déroulée en face à face au mois de mai 2014, par entretiens enregistrés, auprès d'un panel de professionnels sélectionnés selon la méthodologie 
suivante. Des profils d'interlocuteurs permettant d'aboutir à une complémentarité des points de vue et à rendre compte de la multiplicité des points de blocages ont tout d'abord été établis ; il s'agit de personnes occupants des postes stratégiques en matière de production de données et d'analyse de ces dernières (des spécialistes de la qualité de l'air et des chercheurs de disciplines différentes: épidémiologistes, sociologues, spécialistes de la santé publique, spécialistes des inégalités sociales) et en matière de décision (des spécialiste de l'évaluation des politiques publiques, des élus locaux du Grand Lyon/ Rhône-Alpes, des parlementaires). Au total, 14 personnes ont participé à notre enquête. La grille d'entretien a été adaptée en fonction du profil de chaque interlocuteur. Les entretiens enregistrés ont tous été retranscrits. Ces 14 personnes sont réceptives à au moins un volet de cette problématique à savoir : l'environnement, la santé, les politiques publiques et les sciences sociales. Néanmoins aucun de ces interlocuteurs n'a de profil à l'interface de ces différents volets, ce qui pourrait constituer une limite, mais inévitable, lorsque nous nous intéressons à ce type de problématique multidisciplinaire.

\section{Résultats}

\section{Caractérisation du territoire d'étude}

Notre étude a été conduite au sein de l'agglomération de Lyon. La taille de la population concernée (plus d'un millions d'habitants), ses particularités climatiques et géographiques qui rendent la pollution de l'air problématique, son importante activité industrielle (le couloir de la chimie au sud), la distribution socioéconomique de la population sur le territoire ainsi que le volontarisme affiché par les différents acteurs concernés pour agir en faveur de l'environnement font de l'agglomération lyonnaise un terrain d'étude approprié.

19 En ce qui concerne le climat, en hiver, la région lyonnaise est le théâtre d'inversions de températures qui favorisent la stagnation des polluants. Ainsi, les études du PPA 2005-2010 ${ }^{1}$ ont mis en évidence le rôle non négligeable des facteurs météorologiques dans la stagnation et la dispersion de la pollution atmosphérique. En effet, le nord du département est confronté à des situations peu ventées, la dispersion des polluants reste faible; à l'inverse, à l'est et au sud, les conditions de dispersion sont plus favorables en raison des vents forts.

20 L'essentiel de la pollution atmosphérique dans l'agglomération lyonnaise provient des secteurs industriels, des transports routiers ainsi que du secteur résidentiel/tertiaire et de l'activité artisanale ${ }^{2}$. En première position, le dioxyde d'azote est un des polluants majeurs qui est à $63 \%$ d'origine automobile et à $22 \%$ d'origine industrielle ${ }^{3}$. Depuis 1994, les moyennes annuelles mesurées par les stations urbaines sont systématiquement au-dessus de la valeur limite de 2010 établie à $40 \mu \mathrm{g} / \mathrm{m}^{3}$. On trouve ensuite les particules fines dont les principaux secteurs d'activités émetteurs de rejets sont le résidentiel/tertiaire et l'artisanat, le transport routier ainsi que le secteur industriel. Depuis 1995, les moyennes annuelles sont cependant systématiquement inférieures à la valeur limite établie à $40 \mu \mathrm{g} / \mathrm{m}^{3}$ en $2005{ }^{4}$. Il faut également ajouter le fait que la pollution a également un effet amplificateur sur le potentiel allergène des pollens et sur la sensibilité immunologique des muqueuses respiratoires de l'homme. 
L'agglomération lyonnaise a été particulièrement touchée par les pollens de l'ambroisie, très allergènes et largement présents en région Rhône-Alpes.

21 L'agglomération de Lyon présente un dynamisme économique certain : son PIB est de 62 milliards d'euros ( 2 ème place après la ville de Paris et sa région ${ }^{5}$ ). En outre, le revenu moyen de la population lyonnaise est élevé étant donné que la part des diplômés supérieurs y est très importante. En 2007, 15,7\% de la population du Grand Lyon est diplômé d'un niveau Bac +3 et plus, contre $12,1 \%$ pour la moyenne nationale ${ }^{6}$. De récentes études conduites sur l'agglomération de Lyon mettent en évidence l'existence d'un gradient socioéconomique : les IRIS situés dans la partie nord/ouest de l'agglomération de Lyon étant plus favorisés que les IRIS du sud/est (Figure 1).

Des études d'impacts sanitaires réalisées par la cellule Interrégionale d'Epidémiologie de Rhône Alpes ont permis de quantifier l'impact sanitaire de la pollution atmosphérique sur 4 villes de l'est de la France; celles-ci classaient Lyon devant Grenoble, Saint Etienne et Valence en termes de taux de décès anticipés (22/100 000 habitants/an) (Rapport CIRE, 2006).

\section{Analyse des actions territoriales emblématiques menées à Lyon au regard du contexte socioéconomique}

Aujourd'hui, l'analyse de ces différentes actions permet de dénombrer deux types de dispositifs mis en place dans l'agglomération de Lyon qui visent à réduire les niveaux de pollution atmosphérique (tableau 2).

- Dispositif à effet direct : tel que les actions mises en place dans le cadre du dispositif «Mesures exceptionnelles en cas de pic de pollution » visant à diminuer rapidement la pollution de l'air en situation d'urgence (ex. Gratuité des transports en commun - Réduction des vitesses - Circulation des poids lourds interdits dans le tunnel de Fourvière)

- Dispositif à effet indirect : tel que les actions de sensibilisation et d'information du grand public du dispositif «Bulletin Allergo Pollinique» qui vise à communiquer les valeurs relatives aux différents allergènes sur le site internet du Grand Lyon pour permettre aux personnes allergiques de mieux connaitre le risque de survenue de symptômes allergiques (auto prise en charge)

24 Parmi l'ensemble de ces actions, nous avons analysé plus particulièrement une action visant à réduire le trafic automobile dans l'agglomération lyonnaise en favorisant les modes de transports alternatifs à la voiture. Cette action consiste en l'aménagement de plusieurs parkings relais sur le territoire de Lyon. Dans une perspective d'évaluation des effets sanitaires d'une telle action, nous nous sommes intéressés aussi bien aux effets positifs (l'objectif visé) qu'aux effets négatifs, que pourraient engendrer cette action. 
Figure 1 : Distribution spatiale (a) de l'indice socio-économique, (b) des concentrations de $\mathrm{NO}_{2}$ (moyenne globale sur la période 2002 à 2009) dans l'agglomération de Lyon
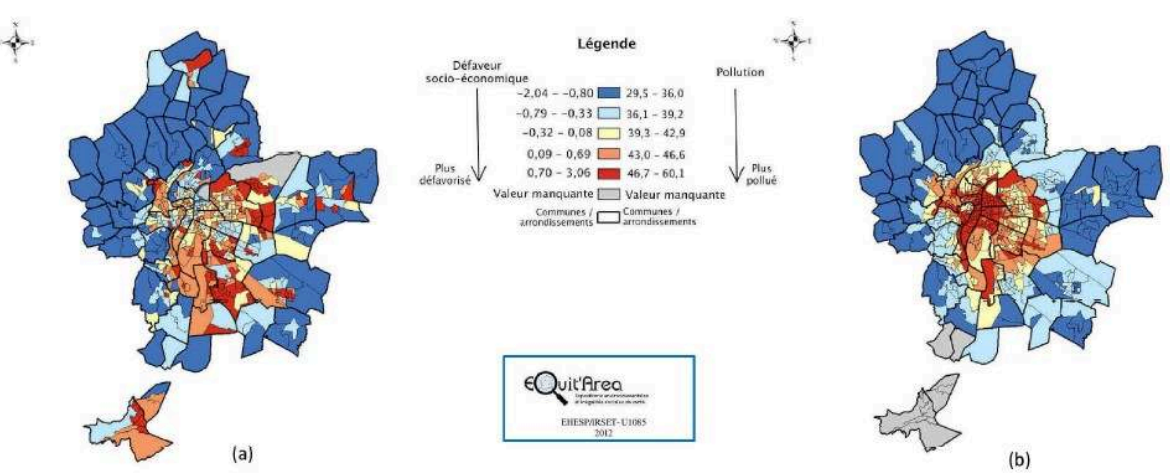

Plus précisément, ces dispositifs ont pour objectif d'inciter les individus à emprunter les autres modes de transport (métro, bus et vélo). Dans cette logique, ces installations permettent :

1. d'une part, de réduire le trafic routier au centre-ville de Lyon, puisqu'on diminue le nombre de véhicules entrant

2. d'autre part, de favoriser la pratique de la marche à pieds des usagers pour aller d'une station de bus/métro à une autre, par exemple.

Ainsi, cet aménagement du territoire améliore la santé de la population générale en diminuant les concentrations des polluants atmosphériques et en augmentant l'activité physique; deux déterminants de santé connus dans la littérature (Projet Aphekom, 2012; Bazex et al., 2012). En effet, l'évaluation des impacts sanitaires de la pollution atmosphérique conduite dans neuf villes françaises (Projet Aphekom, 2012) estime que le gain sanitaire annuel serait de 192,0 décès évitables, de 101,3 décès cardiovasculaires, de 4,4 mois de gain d'espérance de vie à 30 ans pour une réduction de $5 \mu \mathrm{g} /$ $\mathrm{m}^{3}$ des niveaux de particules fines $\left(\mathrm{PM}_{2,5}\right)$ à Lyon. De plus, de nombreuses publications confirment aujourd'hui que la pratique d'activité physique et/ou sportive tout au long de la vie augmente l'espérance de vie en bonne santé, avec une réduction notable de la mortalité prématurée et offre un effet très positif sur le bien-être immédiat, sur l'anxiété et la dépression (Bazex et al., 2012). Cependant, afin de réaliser une évaluation complète des effets sanitaires de l'aménagement de parkings relais, il est important d'intégrer les effets négatifs collatéraux de cette action. En effet, les parkings relais ont été implantés en périphérie de Lyon dans des zones identifiées comme des Contrats Urbains de Cohésion Sociale (CUCS) et plus particulièrement dans les quartiers prioritaires classés CUCS. Avec une capacité de 473 à 18839 Véhicules/jour, ces parkings relais augmenteront le flux de voitures dans ces zones où résident les populations les plus défavorisées (zones classées CUCS 1 et 2 , colorées en rouge et orange sur la Figure 2).

Avec une volonté de réduire le trafic routier au centre de Lyon, cette action territoriale pourrait donc accroitre l'exposition des populations résidant dans les quartiers socialement prioritaires (CUCS 1 ou 2) :

1. à certains polluants de l'air d'origine routière $\left(\mathrm{PM}_{10}, \mathrm{PM}_{2.5}, \mathrm{NO}^{2} \ldots\right)$, principalement 
2. à d'autres nuisances telles que le bruit routier (dû à l'augmentation du flux routier) qui viendrait s'ajouter au bruit du trafic aérien compte tenu de leur proximité à l'aéroport de Lyon.

3. aux accidents de la route, contribuer à détériorer l'état de santé déjà fragile des populations plus défavorisées par cette accumulation de nuisances environnementales, d'autant que sur l'agglomération de Lyon il a été récemment démontré que les effets de la pollution atmosphérique et du bruit sont significativement plus importants chez les populations les plus défavorisées (kihal et al., 2014 ;Padilla et al., 2013; Sexton et al., 1997 ; Rios et al., 2009). pollution atmosphérique sur une zone urbaine pourrait avoir des conséquences sur les ISS. En effet, même lorsque des efforts sont déployés pour réduire les conséquences néfastes de la pollution atmosphérique en un endroit du territoire, des impacts involontairement négatifs directs ou indirects sont observés sur d'autres parties du territoire qui cumulent déjà de multiples difficultés.

\section{Impact des actions territoriales sur les ISS}

Afin de réduire les inégalités sociales de santé, les actions territoriales doivent être à la fois universelles, mais aussi avoir une intensité proportionnelle au désavantage social préexistant. Autrement dit, le choix des priorités doit s'orienter en faveur des catégories de la population qui présentent un cumul de facteurs de risques, ce qui demande un diagnostic sanitaire et social précis de la zone d'étude.

Figure 2 : Illustration de l'évaluation d'une intervention territoriale visant à réduire la pollution de l'air au regard des ISS

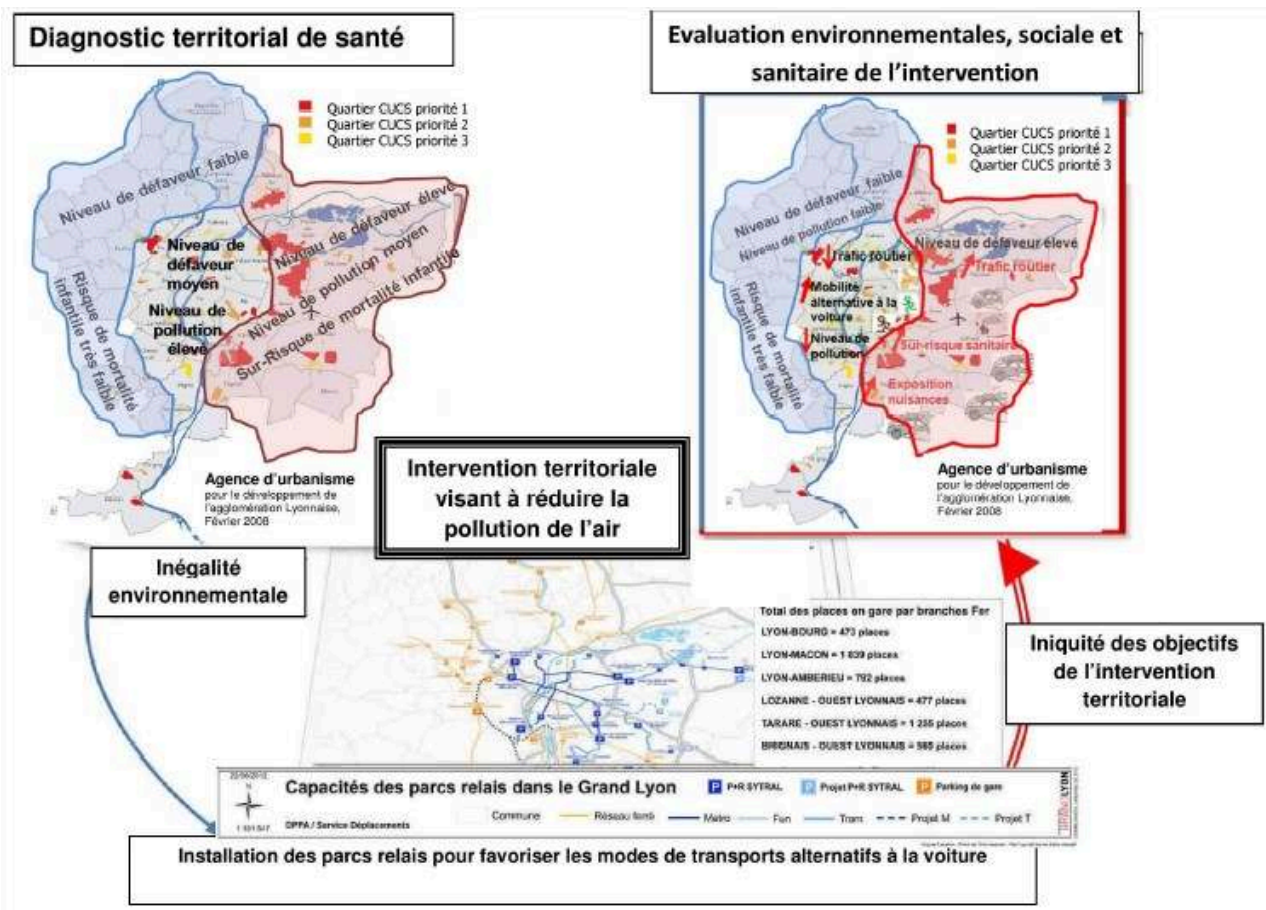

Revue francophone sur la santé et les territoires , Miscellanées 
31 Se pose alors la question de l'évaluation de l'équité en matière de santé des politiques de lutte contre la pollution atmosphérique. Certains professionnels proposent même l'évaluation de l'équité des objectifs des actions territoriales.

Extrait : «Evaluer l'efficacité des actions est déjà une chose complexe. La bonne question est plutôt d'évaluer l'équité des objectifs et pas l'équité des actions en elles-mêmes. Nous avons travaillé sur des communes qui ont mis en place, sans succès, des actions contre le diabète envers les publics défavorisés. La décision était bonne et cohérente avec l'état des lieux des ISS, mais on se rend compte qu'on ne réussit pas toujours à atteindre la cible pour plein de raisons complexes. Après la mise en place d'un dispositif, on se rend compte que certaines personnes ne vont pas utiliser telle ressource, ou tel dispositif. Pour moi, la question de l'équité se pose par rapport aux objectifs (dans quel but a-t-on mis en place l'action, et à destination de quelle population)».

32 A cette question d'évaluation de l'équité en matière de santé des actions territoriales, les différents acteurs et professionnels rencontrés font unanimement état de l'absence de politiques ciblées à l'égard des populations défavorisées en matière de lutte contre la pollution atmosphérique ; c'est ce que nous explique un acteur de la Dreal :

Extrait: «il est évident que les populations qui vont être exposées à des dépassements de normes sont celles qui vont être le plus proche des grands axes structurant de circulation; le périph lyonnais principalement.

Le periph Lyonnais, clairement, c'est pas les belles résidences avec piscine! De plus, avec les vents dominants, c'est déjà naturellement avec un intrant de poussière assez important... On a un gros nœud routier, les industries et effectivement il y a de l'habitation. Et c'est clairement cette tranche de population qui va être exposée.

33 Ce professionnel nous confirme ainsi que certains groupes de population cumulent à Lyon des conditions défavorables à un bon état de santé ; la défaveur socioéconomique et les expositions aux trafics routiers (exposition aux nuisances sonores et aux particules diesel) en se conjuguant augmenteraient la prévalence de certains évènements sanitaires. Les écarts de prévalence de certaines maladies mesurés entre les populations favorisées et défavorisées seraient plus grands.

34 L'absence de prise en compte des ISS dans ces politiques est flagrante dans les outils actuels de planification de l'agglomération lyonnaise (PPA $2{ }^{7}$, programme régional de santé-environnement notamment). Nos entretiens ont confirmé qu'il existe en France encore peu d'études épidémiologiques relatives à la prise en compte des ISS, contrairement à d'autres pays comme le Canada ou les Etats-Unis (Martinez et al., 2002). Nos divers interlocuteurs ont notamment évoqué la complexité d'établir un lien de corrélation entre les pollutions atmosphériques et l'équité des actions de lutte contre ces dernières du fait du caractère multifactoriel de l'état de santé (précarité, nutrition, hygiène...) des populations défavorisées. De plus, il a également été ajouté que cette approche heurtait la conception française républicaine et égalitaire des politiques publiques.

\section{Facteurs influençant le défaut de l'évaluation de l'équité des interventions territoriales}

Le manque d'évaluation des impacts sur les ISS des interventions visant à améliorer la qualité de l'air semble relever d'un problème culturel. Exprimé de diverses façons, les acteurs ont souligné ce problème; un agent de l'ARS rapporte que la réalisation d'évaluations d'impact en santé n'est pas encore ancrée dans notre culture et que 
l'aborder sous l'angle des déterminants sociaux est très rare. Un acteur politique l'aborde autrement :

Extrait «Engager ces questions sous l'angle de la santé, c'est considérer comme pas sérieux, parce que la vraie politique c'est l'économie. Mais il ne faut pas opposer économie et santé ».

En effet, cette thématique est au carrefour d'un ensemble de politiques pour certaines récentes, voire embryonnaires : l'amélioration de la qualité de l'air, l'évaluation des politiques publiques et la réduction des ISS. La prise en compte des ISS dans les politiques environnementales se heurte à un cloisonnement des politiques publiques.

Des initiatives sous l'impulsion d'associations d'élus se sont développées dans le but de favoriser ce décloisonnement comme l'association «Elus, Santé Publique et Territoires ». Pourtant, certains acteurs ont clairement pointé ce frein :

Extrait : « ....problème $:$ le secteur de l'environnement et celui de la santé sont assez cloisonnés... »

Et certains professionnels s'interrogent sur cette limite :

Extrait «On est au début du croisement santé environnementale / ISS avec de la part de certains, une forme d'ignorance..... Je suis surprise de constater que certains experts ne croient pas à la coexistence des deux problématiques santé environnementale / ISS. Je suis inquiète de constater que certains pensent qu'il est inutile, artificiel de vouloir croiser les deux problématiques. »

En effet, les politiques de santé et d'amélioration de la qualité de l'air concernent principalement les ministères de la Santé et de l'Environnement. Cette séparation est visible au niveau des différents types d'acteurs impliqués: plusieurs niveaux de décideurs (national/local, Etat/collectivités territoriales) sont concernés et se confrontent à des expertises parfois plurielles (associations, organismes publics...). Ce cloisonnement concerne également les objectifs de ces politiques : il n'est pas affiché dans les objectifs des politiques d'amélioration de la qualité de l'air de spécifiquement réduire les ISS, mais plutôt de réduire l'exposition de la population sans aucune distinction socioéconomique. De même, en matière d'urbanisme favorable à la santé, les collectivités territoriales disposent d'une autonomie qui leur permet de s'appuyer, ou pas, sur les compétences des différents acteurs dans le domaine des ISS (architecte, urbaniste, ARS, ORS...). Ainsi, le manque de pilotage global et de responsable désigné, concernant l'impact sur les ISS des politiques d'amélioration de la qualité de l'air limite alors l'évaluation sur ce sujet spécifique.

Cependant, même lorsque l'intention de réduire les ISS est affichée dans certaines politiques publiques, on observe souvent une absence d'outils et de systèmes d'information pour conduire et évaluer les politiques permettant de concrétiser cet engagement. En effet, aucune étude d'impact préalable n'est planifiée incluant l'effet prévu sur les ISS des projets d'urbanismes ou des interventions visant à améliorer la qualité de l'air. Les cartographies existantes concernent pour la plupart soit la pollution atmosphérique, soit les ISS, sans effectuer ou prévoir de recoupement de l'information. Enfin, la complexité de ces évaluations n'est compensée par aucune incitation. En effet, les politiques d'amélioration de la qualité de l'air produisent leurs effets sur le long terme et sont donc difficiles à évaluer à courte échéance; ces difficultés ne sont compensées ni par une incitation financière spécifique, ni par une obligation réglementaire particulière. 
Un des acteurs politiques a clairement pointé cette difficulté dans l'adoption des lois en santé publique :

Extrait: «Les gosses asthmatiques, je peux vous dire, c'est les pauvres. Car, les logements sociaux sont au bord du periph; comme les terrains sont moins chers, il y a des logements sociaux, des écoles, des stades, ... c'est scandaleux. ... On fait des budgets à court terme c'est-à-dire pour l'année $\mathrm{n}+1$ et donc les actions de prévention portent ses fruits en année $n+2, n+3$ donc on vous répond, ce que vous proposez coute de l'argent et vous dites que ça va rapporter mais on vous dit oui mais ce n'est pas la même année.... »

«... En matière de santé on a vraiment l'impression de parler dans le désert ... quand je propose de monter des études sur les effets de l'exposition à l'huile de palme ou à l'aspartame et bien on me répond c'est trop cher !»

«En tant que parlementaire, je ne peux pas faire des propositions qui coutent de l'argent. ... Donc, je peux proposer des lois qui ne coutent rien mais qui rapportent de l'argent. ... J'ai beau leur dire que ça coute la première année mais que ça va rapporter la 2ème année »

De plus, la crainte d'une surcharge d'études d'impacts et d'évaluations liée aux politiques que portent les acteurs et le manque de moyens humains sont vraisemblablement des freins majeurs au développement de la pratique de ces évaluations.

\section{Recommandations}

Au niveau Européen, le projet "Determine » a mis en place un processus visant à recenser les politiques qui se sont révélées efficaces pour réduire les inégalités sociales de santé (Projet Determine, 2009). Même si cette initiative n'est pas directement impliquée dans la lutte contre la pollution atmosphérique, ces expériences européennes permettent d'identifier les éléments de succès tout à fait transposables. Plus précisément l'expérience britannique montre aujourd'hui que :

- la définition d'objectifs quantifiés et opérationnels est une étape importante pour la mise en place et l'évaluation de ces différentes interventions.

- la réduction des inégalités sociales de santé appelle des politiques complexes nécessitant de coordonner les actions des différents ministères et les services publics à différentes échelles, du local au national.

\section{Une objectivation des ISS sur un territoire : « une démarche multidisciplinaire »}

Il est nécessaire avant d'introduire l'équité en matière de santé dans les politiques publiques, de formaliser l'objectif de la réduction des ISS sur un territoire. Ainsi, le développement de recherches multidisciplinaires et interventionnelles sera une étape préalable et nécessaire afin i) d'une part, d'enrichir et améliorer les connaissances des ISS, et ii) d'autre part, de passer du constat à l'action.

La mise en place de ce type de recherches, avec le concours des acteurs locaux, permettra :

- La constitution d'une base de données interministérielle croisant des données socioéconomiques, sanitaires et environnementales. 
- Le développement et la production de données à des échelles géographiques fines en passant de la recherche à la production régulière de données statistiques.

- Le développement d'outils d'information et d'analyse des ISS, et les mettre à disposition des décideurs afin d'améliorer la priorisation des actions territoriales.

- Le développement de plateformes intégrées, à l'instar des initiatives de l'Ineris (Caudeville et al., 2011) et du site du projet Equit'Area ${ }^{8}$, conduit à l'École des Hautes Etudes en Santé Publique, pour l'analyse des inégalités environnementales d'exposition avec l'intégration de données de natures différentes (sanitaires, environnementales, socio-économiques)

- De nourrir la réflexion sur des scénarios d'interventions territoriales adaptés à l'objectif de réduction de ces inégalités de santé, et ainsi de proposer des approches transversales aux interventions territoriales.

\section{L'évaluation de l'équité des actions mises en œuvre en matière de santé}

Il est nécessaire, pour prendre en compte les ISS dans les interventions territoriales visant à améliorer la qualité de l'air, d'évaluer, avant leur mise en œuvre, dans quelle mesure l'intervention pourrait accroitre la vulnérabilité ou dégrader d'avantage des conditions de vie des populations socio économiquement défavorisées.

7 Au cours de la dernière décennie sur le plan international et plus récemment en France, l'Evaluation d'Impact sur la Santé (EIS) s'est positionnée en tant que l'outil d'aide à la décision permettant la mise en place de "politiques en matière de santé publique ". Cependant, au-delà des effets sur la santé, même si l'équité en matière de santé est fondamentale dans l'EIS, sa structure ne se prête pas à une action plus ciblée et plus systématique sur ces iniquités (Villeval et al., 2015, Hyndman et al., 2012).

Ainsi, pour prendre efficacement en compte l'équité en matière de santé et plus particulièrement les inégalités de santé dans l'évaluation des interventions territoriales, aujourd'hui, des démarches d'évaluations axées sur l'équité de type d'Evaluation d'Impact sur l'Equité en Santé (EIES) ont été développées avec une attention particulière aux iniquités des déterminants sociaux de la santé. Cette approche systémique actuellement utilisée aux Royaume-Uni (Pays de Galles), en Nouvelle Zélande, en Australie et à l'Ontario, permet de prévoir les effets sanitaires involontaires d'une intervention environnementale territoriale sur des groupes vulnérables ou marginalisés dans la population générale.

La mise en œuvre d'une telle approche dans l'évaluation de l'équité des politiques environnementales nécessitera:

- d'acquérir une véritable culture de l'évaluation des politiques publiques en matière de lutte contre la pollution atmosphérique

- de se questionner sur la faisabilité et l'acceptabilité sociale d'une telle démarche

- l'implication conjointe des décideurs politiques, des acteurs locaux, du citoyen et des chercheurs de différentes disciplines.

- la participation de la population vulnérable concernée par la politique évaluée

- les échanges de données entre les services déconcentrés et les acteurs locaux intervenant dans le domaine des ISS et de l'environnement. 


\section{Conclusion}

50 Aujourd'hui, une question légitime se pose de savoir si les conséquences des interventions luttant contre la pollution de l'air font l'objet d'une évaluation en termes d'équité en matière de santé. C'est en effet grâce à l'évaluation de ces effets qu'il sera à terme possible de prendre efficacement en compte les ISS dans l'élaboration et la mise en œuvre des interventions publiques sur la qualité de l'air comme de l'ensemble des politiques publiques. Conduite sur l'agglomération de Lyon, notre étude confirme le défaut de prise en considération des caractéristiques socioéconomiques des populations dans la réflexion et la priorisation des interventions territoriales mise ou à mettre en place pour lutter contre la pollution atmosphérique. Notre étude souligne également que l'évaluation de l'équité des politiques de lutte contre la pollution atmosphérique reste en France encore principalement du domaine de la recherche. Les acteurs locaux prenant des initiatives nouvelles ou développant des outils innovants, tels que l'évaluation d'impact en santé font figure de pionniers. L'évaluation permet de croiser les regards des différents acteurs, de mettre en œuvre la démocratie participative et, ainsi, de mieux approcher les situations d'injustice environnementale. Afin d'appréhender la complexité des situations d'injustice sanitaire, sociale et environnementale, seule une volonté politique au niveau national pourrait permettre dans une proche échéance de lever les multiples obstacles relevés.

\section{BIBLIOGRAPHIE}

Bazex, Jacques, Pierre Pène, et Daniel Rivière. 2012. « [Physical activities and sport; implications for health and society] ». Bulletin De l'Académie Nationale De Médecine 196 (7): 142942.

Benmarhnia T, Rey L, Cartier Y, Clary CM, et al. 2014. Addressing equity in interventions to reduce air pollution in urban areas: a systematic review. Int J Public Health. 59(6):933-44

Caudeville J., 2011. Développement d'une plateforme intégrée pour la cartographie de l'exposition des populations aux substances chimiques : construction d'indicateurs spatialisés en vu d'identifier les inégalités environnementales à l'échelle régionale. Compiègne : Université Technologique de Compiègne, $198 \mathrm{p}$.

Centre d'analyse stratégique-« Lutte contre l'obésité : repenser les stratégies préventives en matière d'information et d'éducation ", Note de veille, mars 2010. Document disponible en ligne : http://oullier.free.fr/files/2010_Sauneron-Gimbert-Oullier_NoteVeilleCAS_ObesiteNeuroscience-Genetique-Surpoids.pdf

DETERMINE and EU Consortium for action on the socio-economic determinants of Health: a report. 2009. En ligne : http://ec.europa.eu/health/ph_determinants/socio_economics/ cons_inequities_en.htm.in http://www.Health-inequalities.eu

Declercq C, Pascal M, Chanel O, Corso M, Ung A et al. 2012. Impact sanitaire de la pollution atmosphérique dans neuf villes françaises. Résultats du projet Aphekom. Saint-Maurice: Institut de veille sanitaire. $33 \mathrm{p}$ 
Deguen S, Zmirou-Navier D., 2010. Social inequalities resulting from health risks related to ambient air quality-A European review. European Journa of Public Health.Janv 2010; 20(1):27-35

Havard S, Deguen S, Zmirou-Navier D, et al. 2009. Traffic-related air pollution and socioeconomic status: a spatial autocorrelation study to assess environmental equity on a small-area scale. Epidemiology, 20(2): 223-230

Hyndman et al., 2012. Guide de travail pour l'évaluation de l'impact sur l'équité en matière de santé (EIES)Document de travail élaboré par la ministère de la Santé et des Soins de longue durée (MSSLD) en partenariat avec le secteur de la santé publique et les fournisseurs de services de santé. Accessible en ligne : http://www.health.gov.on.ca/fr/pro/programs/heia/docs/ workbook.pdf

Kihal-Talantikite W, Padilla CM, Lalloue B, Rougier C, Defrance J, Zmirou-Navier D, Deguen S., 2013. An exploratory spatial analysis to assess the relationship between deprivation, noise and infant mortality.Environmental Health, 12:109

Le Blanc G, 2009. L'invisibilité sociale, Paris, PUF, 208PP. Collection "Pratiques théoriques" $\mathrm{N}^{\circ} 1$. ISBN : 978-2-13-057347-0

Martinez-Alier J, 2002. The environmentalism of the poor, A study of ecological conflicts and evaluation. Document accessible en ligne : http://web.boun.edu.tr/ali.saysel/ESc307/AlierCurrents of Environmentalism.pdf

O'Neill MS, Jerrett M, Kawachi I, et al. 2003. Health, wealth, and air pollution: advancing theory and methods. Environ Health Perspect; 111(16):1861-70.

Padilla CM, Deguen S, Lalloue B, Blanchard O, Beaugard C, Troude F, Navier DZ, Vieira VM. 2013a. Cluster analysis of social and environment inequalities of infant mortality. A spatial study in small areas revealed by local disease mapping in France.Sci Total Environ. 1; 0: 433-441.

Rapport du groupe d'étude AFSSET, Septembre 2007, Etude d'impact sur les coûts que représentent pour l'Assurance maladie certaines pathologies liées à la pollution.

Rapport du HCSP 2013 : Évaluation du deuxième Plan national Santé-Environnement : rapport du Haut Conseil de la santé publique, Sep 2013.

Rapport CIRE-Rhône-Alpes, 2006. Evaluation de l'impact de la pollution atmosphérique urbaine sur l'agglomération de Lyon. Document accessible en ligne : http://www.invs.sante.fr/ publications/2006/pollution_lyon/rapport_pollution_lyon.pdf

Rios R, Poje GV, Detels R. 1993. Susceptibility to environmental pollutants among minorities.ToxicolInd Health, 9(5): 797-820.

Sexton K. 1997. Sociodemographic aspects of human susceptibility to toxic chemicals: Do class and race matter for realistic risk assessment? Environmental Toxicology and Pharmacology, 4(3-4): 261-9.

Villeval, Mélanie, Elsa Bidault, Thierry Lang, et pour le groupe AAPRISS. 2015. « Evaluation D'impact Sur La Santé et Évaluation D'impact Sur L'équité En Santé : Éventail de Pratiques et Questions de Recherche ». Global Health Promotion, doi:10.1177/1757975915570139.

\section{ANNEXES}




\section{Annexe}

Tableau 1 : Recensement des mesures les plus emblématiques mises en place sur le Grand Lyon pour lutter contre la pollution atmosphérique

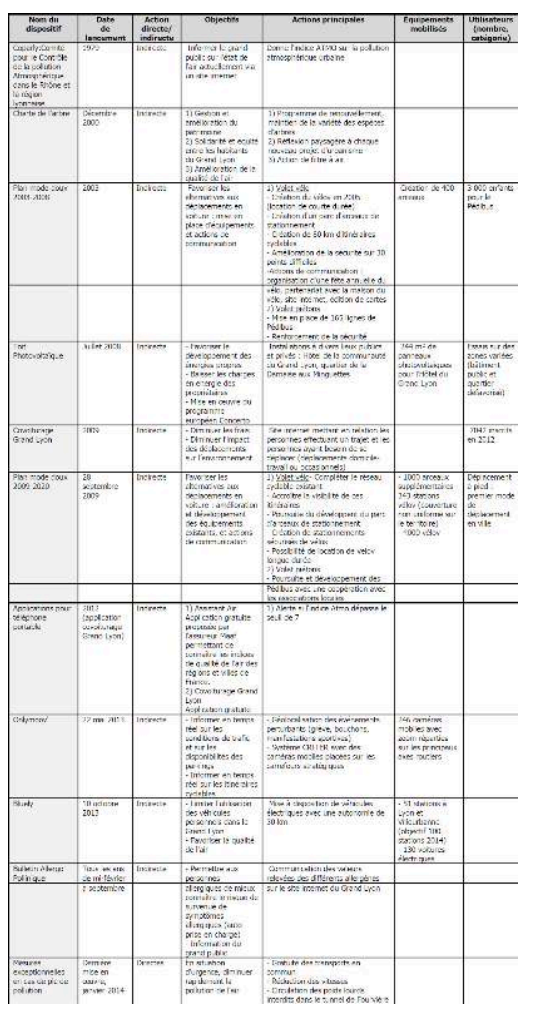

\section{NOTES}

1. Plan de Protection de l'Atmosphère de l'agglomération lyonnaise : http://www.ppalyon.org

2. Plan de Protection de l'Atmosphère de l'agglomération lyonnaise (objectif 2010), p. 68

3. Ibid, P68

4. Plan de Protection de l'Atmosphère de l'agglomération lyonnaise (objectif 2010), p. 68

5. « lyon-business.org - Chiffres-clés sur Lyon et sa région ».

6. Observatoire du développement durable du Grand Lyon. Référentiel social 2011, p. 5.

7. PPA 2 approuvé en 2014 qui révise le PPA 1.

8. http://www.equitarea.org 


\section{RÉSUMÉS}

La combinaison de la pollution atmosphérique et de la défaveur socioéconomique peut contribuer à l'aggravation des inégalités de santé. De nombreuses interventions de lutte contre la pollution atmosphérique sont mises en place à différentes échelles du territoire. La question soulevée est la suivante : "les bénéfices sur la santé des interventions visant à améliorer la qualité de l'air different-ils selon les caractéristiques socioéconomiques des populations? ". Un panel de professionnels et d'acteurs politiques a été interrogé sur l'enjeu de l'équité en matière de santé dans l'élaboration et la mise en œuvre de ces interventions. Cette recherche révèle une absence d'évaluation de l'équité des politiques environnementales. Afin de prendre en compte les inégalités sociales de santé, nous suggérons d'évaluer systématiquement dans quelle mesure les interventions pourraient involontairement accroitre la vulnérabilité des populations défavorisées par une approche de type Evaluation d'Impact sur l'Equité en matière de Santé.

Background - The relationship between the characteristics of the neighbourhoods where people live and health inequalities is now well documented. More specifically, socioeconomic characteristics have been indicated as mediating the health effect of environmental exposure; this is most documented in relation to air pollution. Two mechanisms have been advanced to explain the possible combined effect of socioeconomic status and environmental exposure: i) differential exposure, which explores the possibility that environmental pollutants are not equally distributed across population groups with different socioeconomic characteristics; ii) differential vulnerability, which states that, at the same level of exposure, socially disadvantaged groups experience more severe health effects.

Since the '70s, corresponding to a growth in awareness of environmental issues, the number of interventions to protect the state of the environment in Europe increased. In France, there are many local interventions which aim to improve air quality, such as the bike share system, the French "bonus / malus" system, areas with $30 \mathrm{~km} / \mathrm{h}$ speed limit and greater attention to environmental issues in urban planning. However, who will really benefit from these local interventions?

objective - The question raised in this present article is "Are the health benefits of interventions which aim to reduce air pollution, experienced equally between populations characterized by different socioeconomic status? “

Material and methods - Our study is conducted in Lyon Metropolitan area located in Eastern France. We questioned a sample of 14 professionals with contrasting profiles in terms of their environmental engagement (such as decision-makers, specialists of public policies evaluation and researchers). We focused our interview on three points: the actions and the public policies implemented to decrease the atmospheric pollution; the assessment of these actions in term of health benefits and impact on the social health inequalities; recommendations to improve the consideration of equity in public health interventions.

Our article is structured as follows. We will describe, in the first section, the Lyon Metropolitan area (Lyon MA). In the second section, we will give a brief summary of the principal interventions related to air pollution implemented in Lyon MA. In this section, we will also detail the consequences of one specific intervention in relation to neighborhood socioeconomic characteristics. In the third section, we will discuss the factors which might influence the lack of an assessment of implications for health equity in local interventions. Finally, the last section suggests recommendations for future investigations.

Results - All professionals reported the absence of an assessment of health inequity as part of intervention; they added that, to their knowledge, social characteristics are not systematically 
reviewed as part of public health policy interventions. In France, the political climate could explain the lack of attention to issues of heath equity and social health inequity within local action planning; additionally, those professionals interviewed noticed a clear separation between heath, environment, and urbanism policies. Exceptions are found in public health research where socioeconomic status is considered as one important characteristic in health assessment of interventions aiming to improve air quality.

Conclusion - The principal recommendation is to include equity issues at the beginning of the development of any given intervention within a territory; the underlying idea is to take into account in the process the ways in which the impact of such an intervention could be socially differentiated. This concept has already been introduced in several countries, for example in Canada, through the approach of Health Equity Impact Assessment (HEIA). In France, it is time to move from the health impact assessment to the HEIA in order to maximize the positive impacts of interventions and to reduce the collateral negative effects that could potentially widen health disparities between population groups.

\section{INDEX}

Mots-clés : pollution atmosphérique, pollution, inégalités sociales et territoriales de santé, équité, pratiques professionnelles, interventions

\section{AUTEURS}

\section{WAHIDA KIHAL}

Ingénieur de Recherche au département DSET\&GS, EHESP School of Public Health, INSERM U1085 (IRSET), Rennes, France

\section{CINDY PADILLA}

Enseignante-chercheuse au département Epi-Biostat, EHESP School of Public Health, Rennes, France

\section{SÉVERINE DEGUEN}

Enseignante-chercheuse au département DSET\&GS, EHESP School of Public Health, INSERM U1085 (IRSET), Rennes, France 